\title{
Estimation of the integral of a stochastic process
}

\section{Noel Cressie}

Consider the class of stochastic processes with stationary independent increments and finite variances; notable members are brownian motion, and the Poisson process. Now for $x_{t}$ any member of this class of processes, we wish to find the optimum sampling points of $X_{t}$, for predicting $\int_{0}^{A} x_{t} d t$. This design question is shown to be directly related to finding sampling points of $Y_{t}$ for estimating $B$ in the regression equation, $y_{t}=\beta t^{2}+X_{t}$. Since processes with stationary independent increments have linear drift, the regression equation for $y_{t}$ is the first type of departure we might look for; namely quadratic drift, and unchanged covariance structure.

\section{Introduction}

Statistical inference on stochastic processes, in particular for problems of design, is an area of research which has been neglected until recently. In this paper we concentrate on processes $X_{t}$, with stationary independent increments, with finite variances, and with $X_{0^{\circ}}=0$; this includes both the brownian motion, and the Poisson process as special cases. Define $I$ to be

Received 2 November 1977. The author gives due acknowledgement to F. Samaniego, who originally posed this problem.

83 


$$
I \equiv \int_{0}^{A} x d t
$$

which is well defined as the limit in quadratic mean of the approximating Riemann sums. We are interested in the design problem of predicting this integral, based on observing the process at $n$ distinct time points,

$$
0<t_{1}<t_{2}<\ldots<t_{n} \leq A \text {. }
$$

Sacks and YIvisaker [1] studied this problem for a large class of random processes; we show here that for processes with stationary independent increments, and finite variance, the design points can be found explicitly.

The motivation for considering the prediction of $\int_{0}^{A} X_{t} d t$ was given by Sacks and YIvisaker. They showed that the problem arose naturally from the following interesting question. Let $y_{t}$ be a stochastic process with $y_{0}=0$. Now suppose $y_{t}$ can be regressed on a deterministic function $f(t)$, with $X_{t}$ as error; that is

$$
y_{t}=\beta f(t)+X_{t}, \quad 0<t \leq A
$$

Let $R(s, t)$ be the covariance function of $X_{t}$, and suppose

$$
f(t)=\int_{0}^{A} R(s, t) \phi(s) d s
$$

$\phi$ continuous. Then finding the design that minimizes the variance of $\sum z_{i} y_{i}$, the best linear unbiased estimator of $\beta$ based on $\mathbf{t}=\left(t_{1}, \ldots, t_{n}\right)$, can be shown to be equivalent to finding the optimal (minimum mean squared error) design to predict $\int_{0}^{A} \phi(t) X_{t} d t$.

For our case $\phi(t) \equiv I$, and we will see below that $R(s, t)=\sigma^{2} \cdot \min (s, t)$. Hence the regression equation becomes

$$
y_{t}=\beta^{\prime} t^{2}+X_{t}^{\prime}
$$


where $\beta^{\prime}=-\beta \sigma^{2} / 2$, and $X_{t}^{\prime}=X_{t}+\left(A B \sigma^{2}\right) t$, which is still a process with stationary independent increments. In general, $X_{t}^{\prime}$ has a drift proportional to $t$ (see below). Clearly then, adding the quadratic term $\beta^{\prime} t^{2}$ to the drift is the first type of departure (from brownian motion for example) we might seek for the process $Y_{t}$. Hence finding the right design to estimate $B$ is of primary importance. Subsequently, our interest will be in the equivalent problem of finding the optimal design to estimate $\int_{0}^{A} x_{t} d t$.

Define $t \equiv\left(t_{1}, \ldots, t_{n}\right)$. Using minimum mean squared error as the optimality criterion, the optimal predictor becomes

$$
\begin{aligned}
\hat{I}_{t} & =E\left(I \mid x_{t_{1}}, \ldots, x_{t_{n}}\right) \\
& =\int_{0}^{A} E\left(x_{t} \mid x_{t_{1}}, \ldots, x_{t_{n}}\right) d t .
\end{aligned}
$$

When $x_{t}$ has stationary independent increments, $E\left(x_{t} \mid x_{t_{1}}, \ldots, x_{t_{n}}\right)$ may be shown to be the polygon obtained by linearly connecting the points $(0,0),\left(t_{1}, x_{t_{1}}\right), \ldots,\left(t_{n}, x_{t_{n}}\right) ;$ and for $t>t_{n}$, we have a straight line from $\left(t_{n}, X_{t_{n}}\right)$ with slope $m$, where $m=E\left(X_{1}\right)$.

Also, the Levvy representation of the characteristic function of $X_{t}$ tells us that

$$
E\left(X_{t} X_{s}\right)=s\left(\sigma^{2}+m^{2} t\right), s<t
$$

where

$$
\sigma^{2}=\frac{\operatorname{var}\left(X_{t}\right)}{t} \text { and } m=\frac{E\left(X_{t}\right)}{t} \text {. }
$$

Therefore, $\operatorname{cov}\left(x_{t}, x_{s}\right)=\sigma^{2} \cdot \min (s, t)$.

In what is to follow, Sections 2 and 3 completely solve the design problem as posed above. Then Section 4 extends the problem to prediction 
of $\int_{u}^{v} x_{t} d t, \quad 0<u<v \leq A ;$ again the optimal design can be found explicitly.

\section{To calculate the mean squared error}

Define

$$
\operatorname{MSE}_{\mathrm{t}} \equiv E\left[\left(I-\hat{I}_{\mathrm{t}}\right)^{2}\right]
$$

where $I$ and $\hat{I}_{t}$ are given in (1) and (2). Our task is to find the $t$ which will minimize $\mathrm{MSE}_{\mathrm{t}}$. Firstly, we will show that without loss of generality, we may consider $m=0$.

Define $z_{t} \equiv X_{t}-m t$; then $E\left(z_{t}\right)=0$, for all $t \geq 0$, and

$$
\begin{aligned}
E\left(x_{t} \mid x_{t_{1}}, \ldots, x_{t_{n}}\right) & =E\left(x_{t} \mid z_{t_{1}}, \ldots, z_{t_{n}}\right) \\
& =E\left(z_{t} \mid z_{t_{1}}, \ldots, z_{t_{n}}\right)+m t .
\end{aligned}
$$

Therefore,

$$
x_{t}-E\left(x_{t} \mid x_{t_{1}}, \ldots, x_{t}\right)=z_{t}-E\left(z_{t} \mid z_{t_{1}}, \ldots, z_{t_{n}}\right) .
$$

Hence we can do all our calculations on $\mathrm{MSE}_{t}$ as if $m=0$; that is as if

(4)

$$
E\left(x_{t}\right)=0
$$

$$
E\left(X_{t} X_{s}\right)=\sigma^{2} \cdot \min (s, t) \text {. }
$$

We will also show that $\mathrm{MSE}_{\mathbf{t}}$ can be partitioned into a sum of squares of orthogonal components. Consider, with $a<b<c$, 


$$
\begin{aligned}
\left.E \iint_{a}^{c}\left[X_{t}-E\left(x_{t} \mid x_{a}, x_{b}, x_{c}\right)\right] d t\right)^{2} & \\
& =E\left[\int_{a}^{b}\left[x_{t}-E\left(x_{t} \mid x_{a}, x_{b}\right)\right] d t+\int_{b}^{c}\left[x_{t}-E\left(x_{t} \mid x_{b}, x_{c}\right)\right] d t\right)^{2} \\
& =E\left(\int_{a}^{b}\left[x_{t}-E\left(x_{t} \mid x_{a}, x_{b}\right)\right] d t\right)^{2}+E\left(\int_{b}^{c}\left[x_{t}-E\left(x_{t} \mid x_{b}, x_{c}\right)\right] d t\right)^{2} .
\end{aligned}
$$

This is because

$$
\begin{aligned}
& E\left(\int_{a}^{b}\left[X_{t}-E\left(X_{t} \mid X_{a}, X_{b}\right)\right] d t \cdot \int_{b}^{c}\left[X_{s}-E\left(X_{s} \mid x_{b}, X_{c}\right)\right] d s\right) \\
&=\int_{b}^{c} \int_{a}^{b} E\left[\left[X_{t}-\frac{X_{b}-X_{a}}{b-a}(t-a)-X_{a}\right]\left[X_{s}-\frac{X_{c}-X_{b}}{c-b}(s-b)-X_{b}\right]\right) d t d s \\
&=\sigma^{2} \int_{b}^{c} \int_{a}^{b} 0 \\
&=0 .
\end{aligned}
$$

Therefore, using the identical argument just given, we may write

$$
\text { (5) } \begin{aligned}
\mathrm{MSE}_{t}=E\left(\int_{0}^{t_{1}}\left[x_{t}-E\left(x_{t} \mid x_{t_{1}}\right)\right] d t\right)^{2} & +E\left(\int_{t_{1}}^{t_{2}}\left[x_{t}-E\left(x_{t} \mid x_{t_{1}}, x_{t_{2}}\right)\right] d t\right)^{2} \\
& +\ldots+E\left[\int_{t_{n-1}}^{t_{n}}\left[x_{t}-E\left(x_{t} \mid x_{t_{n-1}}, x_{t_{n}}\right)\right] d t\right)^{2} \\
& +E\left[\int_{T_{n}}^{A}\left[x_{t}-E\left(x_{t} \mid x_{t_{n}}\right)\right] d t\right)^{2}
\end{aligned}
$$

We will now calculate the contribution of each of these terms to $\mathrm{MSE}_{t}$. Consider 


$$
\begin{aligned}
& E\left\{\int_{a}^{b}\left[X_{t}-E\left(x_{t} \mid x_{a}, x_{b}\right)\right] d t\right)^{2} \\
& \quad=E\left(\int_{a}^{b} x_{t} d t-\frac{\left(x_{b}+X_{a}\right)}{2}(b-a)\right]^{2} \\
& \quad=E\left(2 \int_{a}^{b} \int_{a}^{t} x_{s} x_{t} d s d t\right)-2 E\left(\frac{\left(x_{b}+X_{a}\right)}{2}(b-a) \int_{a}^{b} x_{t} d t\right)+E\left(\frac{\left(x_{b}+X_{a}\right)}{2}(b-a)\right)^{2},
\end{aligned}
$$

which, by repeated usage of (4), is equal to

$$
\frac{\sigma^{2}(b-a)^{3}}{12}
$$

The end interval $\left(t_{n}, A\right)$ is a special case. Using similar arguments to the above, we may obtain

$$
E\left(\int_{t_{n}}^{A}\left[X_{t}-E\left(X_{t} \mid X_{t_{n}}\right)\right] d t\right)^{2}=\frac{\left.\sigma^{2}(A-T)_{n}\right)^{3}}{3} .
$$

Therefore, (5), (6), and (7) give us

$$
\mathrm{MSE}_{t}=\sigma^{2}\left[\sum_{i=0}^{n-1} \frac{\left(t_{i+1}-t_{i}\right)^{3}}{12}+\frac{\left(A-t_{n}\right)^{3}}{3}\right] \text {, }
$$

where $t_{0} \equiv 0$.

3. Minimizing the mean squared error We will now minimize expression (8), by putting

$$
\frac{\partial}{\partial t_{i}} \mathrm{MSE}_{t}=0, i=1, \ldots, n \text {. }
$$

This gives us the following $n$ equations:

$$
t_{i}=\frac{t_{i+1}+t_{i-1}}{2}, i=1, \ldots, n-1,
$$

$$
t_{n}=\frac{2 A}{3}+\frac{t_{n-1}}{3} \text {. }
$$

Solving (9) we get 


$$
t_{j}=\frac{j}{2} t_{2}, j=1, \ldots, n,
$$

and putting this into $(10)$ allows us to solve for $t_{2}$. Hence the solution to (9) and (10) is

$$
t_{i}^{0}=\frac{2 i}{2 n+1} A, \quad i=1, \ldots, n
$$

Substituting (11) into ( 8 ), we get

$$
\mathrm{MSE}_{t^{0}}=\frac{\sigma^{2} A^{3}}{3(2 n+1)^{2}},
$$

which can be shown to be greater than or equal to $\mathrm{MSE}_{t}$ for all $t$. Therefore, choosing $t=t^{0}$ gives us the optimal design.

\section{Optimal design for predicting $\int_{u}^{v} x_{t} d t$}

We now wish to find the optimal design for predicting $\int_{u}^{v} x_{t} d t$, where $0<u<v \leq A$. Recall that our sampling points $t_{1}, t_{2}, \ldots, t_{n}$ are such that $0<t_{1}<t_{2}<\ldots<t_{n} \leq A$. Since $X_{t}$ has stationary independent increments, common sense tells us that we are going to want to sample all our points in $[u, v]$. To show that this is in fact true, suppose that we have

$$
t_{j-1} \in[0, u]
$$

and

$$
t_{k+1} \in[v, A],
$$

where $2 \leq j \leq k \leq n-1$. Define 


$$
\begin{aligned}
s_{t} \equiv E\left[\int_{u}^{v}\left[x_{t}-E\left(x_{t} \mid x_{t_{1}}, \ldots, x_{t}\right)\right] d t\right)^{2} \\
=E\left\{\int_{u}^{t} j\left[x_{t}-E\left(x_{t} \mid x_{t_{j-1}}, x_{t_{j}}\right)\right] d t\right)^{2} \\
+E\left\{\int_{t_{j}}^{t}\left[x_{t}-E\left(x_{t} \mid x_{t_{j}}, x_{t_{j+1}}\right)\right] d t\right)^{2} \\
+\ldots+E\left[\int_{t_{k}}^{v}\left[x_{t}-E\left(x_{t} \mid x_{t_{k}}, x_{t_{k+1}}\right)\right] d t\right)^{2} .
\end{aligned}
$$

The middle contributions to $S_{t}$ are, as before,

$$
\frac{\sigma^{2}\left(t_{i+1}-t_{i}\right)^{3}}{12}, \quad i=j, j+1, \ldots, k-1 .
$$

The end contributions can be found by a method almost identical to the method used to obtain (6). They are

$$
\sigma^{2}\left\{\frac{\left(t_{j}-u\right)^{3}}{3}-\frac{1}{4} \frac{\left(t_{j}-u\right)^{4}}{t_{j} t_{j-1}}\right\}, t_{j-1} \in[0, u],
$$

and

$$
\sigma^{2}\left\{\frac{\left(v-t_{k}\right)^{3}}{3}-\frac{1}{4} \frac{\left(v-t_{k}\right)^{4}}{t_{k+1}-t_{k}}\right\}, t_{k+1} \in[v, A] .
$$

Obviously, over all choices of $t_{j-1}$ and $t_{k+1}$, the one which makes $s_{t}$ the smallest is

$$
t_{j-1}=u \text { and } t_{k+1}=v
$$

In other words, if we restrict $t_{j-1}$ to belong to $[0, u]$, then the best choice of $t_{j-1}$ is $u$, and similarly, if we restrict $t_{k+1}$ to belong to $[v, A]$, then the best choice of $t_{k+1}$ is $v$.

Common sense also tells us that a design with $j$ as small as possible (allowing more points to be sampled in $[u, v]$ ), that is, with 
$j=2, k=n-1$, will have smaller mean squared error. This can also be verified, with a little algebra.

Let us now try to minimize $s_{t}$ for $t$ satisfying $u \leq t_{1}<t_{2}<\ldots<t_{n} \leq v$.

$$
\begin{array}{r}
s_{t}=E\left(\int_{u}^{t}\left|x_{t}-E\left(x_{t} \mid x_{t_{1}}\right)\right| d t\right\}^{2}+E\left\{\int_{t_{1}}^{t_{2}}\left|x_{t}-E\left(x_{t} \mid x_{t_{1}}, x_{t_{2}}\right)\right| d t\right)^{2} \\
+\ldots+E\left(\int_{t_{n}}^{v}\left|x_{t}-E\left(x_{t} \mid x_{t_{n}}\right)\right| d t\right)^{2} .
\end{array}
$$

As before, the middle contributions are

$$
\frac{\sigma^{2}\left(t_{i+1}-t_{i}\right)^{3}}{12}, i=1,2, \ldots, n-1,
$$

and from (7) the last term is

$$
\frac{\sigma^{2}\left(v-t_{n}\right)^{3}}{3}
$$

The first term is derived using the method identical to the one used to get (6) and is equal to

$$
\sigma^{2}\left[\frac{t_{1}^{3}-u^{3}}{3}-u^{2}\left(t_{1}-u\right)-\frac{1}{4} \frac{\left(t_{1}^{2}-u^{2}\right)^{2}}{t_{1}}\right]
$$

Add together (12), (13), (14) to give $s_{t}$, and then set

$$
\frac{\partial}{\partial t_{i}} s_{t}=0, i=1, \ldots, n \text {. }
$$

This results in the following set of $n$ equations:

$$
\begin{aligned}
& t_{2}=2 t_{1}-u^{2} / t_{1}, \\
& t_{i}=\frac{t_{i+1}+t_{i-1}}{2}, i=2, \ldots, n-1, \\
& t_{n}=\frac{2 v}{3}+\frac{t_{n-1}}{3} .
\end{aligned}
$$


Solving (15) recursively gives

$$
t_{1}^{0}=\frac{v+\sqrt{v^{2}+\left(4 n^{2}-1\right) u^{2}}}{2 n+1}
$$

and

$$
t_{j}^{0}=j t_{1}^{0}-\frac{(j-1) u^{2}}{t_{1}^{0}}, j=1, \ldots, n
$$

From equation (16),

$$
u^{2}=\frac{\left[t_{1}^{0}\right]^{2}(2 n+1)-2 v t_{1}^{0}}{(2 n-1)}
$$

and putting this into (17) gives

$$
t_{j}^{0}=\frac{2(j-1) v}{2 n-1}+\left[\frac{2 n-2 j+1}{2 n-1}\right] t_{1}^{0}, j=1, \ldots, n,
$$

resulting in mean squared error

$$
s_{t^{0}}=\sigma^{2}\left[\frac{\left[t_{1}^{0}\right)^{3}}{12}-\frac{u^{2} t_{1}^{0}}{2}+\frac{2}{3} u^{3}-\frac{u^{4}}{4 t_{1}^{0}}\right]+\frac{\sigma^{2}\left(v-t_{1}^{0}\right)^{3}}{3(2 n-1)^{2}} .
$$

Therefore

$$
\begin{aligned}
s_{\mathbf{t}^{0}} & =\min \left\{S_{\mathbf{t}}: u \leq t_{1}, t_{n} \leq v\right\} \\
& \leq \min \left\{S_{\mathrm{t}}: t_{1}=u, t_{n}=v\right\} \\
& =\min \left\{S_{\mathrm{t}}: t_{1} \in[0, u], t_{n} \in[v, A]\right\} \\
& \leq \min \left\{S_{\mathrm{t}}: t_{j-1} \in[0, u], t_{k+1} \in[v, A]\right\}, 2 \leq j \leq k \leq n-1,
\end{aligned}
$$

that is $S_{t^{0}} \leq S_{t}$, for all $t \in[0, A]^{n}$. Hence the optimal design for predicting $\int_{u}^{v} x_{t} d t$, where $x_{t}$ is any process with stationary independent increments, is given by (16) and (18). 


\section{Reference}

[1] Jerome Sacks and Donald YIvisaker, "Statistical designs and integral approximation", Time series and stochastic processes; convexity and combinatorics, 115-136 (Proc. Twelfth Biennial Seminar, Canadian Mathematical Congress, University of British Columbia, 1969. Canadian Mathematical Congress, Société Mathématique du Canada, Montreal, Canada, 1970).

School of Mathematical Sciences,

Flinders University,

Bedford Park,

South Australia. 